\title{
High throughput sequencing identifies an imprinted gene, Grb10, associated with the pluripotency state in nuclear transfer embryonic stem cells
}

\author{
Hui Li, ${ }^{1,2,3}$, Shuai Gao ${ }^{3}$, Hua Huang ${ }^{5}$, Wenqiang Liu ${ }^{4}$, Huanwei Huang ${ }^{3}$, Xiaoyu Liu ${ }^{4}$, \\ Yawei Gao ${ }^{4}$, Rongrong Le ${ }^{4}$, Xiaochen Kou ${ }^{4}$, Yanhong Zhao ${ }^{4}$, Zhaohui Kou ${ }^{3}$, Jia Li ${ }^{3}$, \\ Hong Wang ${ }^{4}$, Yu Zhang ${ }^{3}$, Hailin Wang ${ }^{2,5}$, Tao Cai ${ }^{3}$, Qingyuan Sun ${ }^{1,2}$, Shaorong Gao ${ }^{4}$ \\ and Zhiming Han ${ }^{1}$ \\ ${ }^{1}$ State Key Laboratory of Stem Cell and Reproductive Biology, Institute of Zoology, Chinese Academy of Sciences, Beijing, \\ People's Republic of China \\ ${ }^{2}$ University of Chinese Academy of Sciences, Chinese Academy of Science, Beijing, People's Republic of China \\ ${ }^{3}$ National Institute of Biological Sciences, NIBS, Beijing, People's Republic of China \\ ${ }^{4}$ Clinical and Translational Research Center of Shanghai First Maternity \& Infant Hospital, School of Life Sciences and \\ Technology, Tongji University, Shanghai, People's Republic of China \\ ${ }^{5}$ State Key Laboratory of Environment Chemistry and Ecotoxicology, Research Center for Eco-Environmental Sciences, \\ Chinese Academy of Science, Beijing, People's Republic of China \\ Correspondence to: Zhiming Han, email: hanzm@ioz.ac.cn \\ Shaorong Gao, email: gaoshaorong@tongji.edu.cn \\ Keywords: nuclear transfer reprogramming, induced pluripotent reprogramming, DIk 1-Dio3 region, imprinted gene, RNA-seq \\ Received: February 10, $2017 \quad$ Accepted: March 24, $2017 \quad$ Published: April 18, 2017 \\ Copyright: Li et al. This is an open-access article distributed under the terms of the Creative Commons Attribution License 3.0 (CC BY 3.0), \\ which permits unrestricted use, distribution, and reproduction in any medium, provided the original author and source are credited.
}

\section{ABSTRACT}

Somatic cell nuclear transfer and transcription factor mediated reprogramming are two widely used techniques for somatic cell reprogramming. Both fully reprogrammed nuclear transfer embryonic stem cells and induced pluripotent stem cells hold potential for regenerative medicine, and evaluation of the stem cell pluripotency state is crucial for these applications. Previous reports have shown that the DIk1-Dio3 region is associated with pluripotency in induced pluripotent stem cells and the incomplete somatic cell reprogramming causes abnormally elevated levels of genomic 5-methylcytosine in induced pluripotent stem cells compared to nuclear transfer embryonic stem cells and embryonic stem cells. In this study, we compared pluripotency associated genes Rian and Gt/2 in the Dlk1-Dio3 region in exactly syngeneic nuclear transfer embryonic stem cells and induced pluripotent stem cells with same genomic insertion. We also assessed 5-methylcytosine and 5-hydroxymethylcytosine levels and performed highthroughput sequencing in these cells. Our results showed that Rian and Gt/2 in the DIk1Dio3 region related to pluripotency in induced pluripotent stem cells did not correlate with the genes in nuclear transfer embryonic stem cells, and no significant difference in 5-methylcytosine and 5-hydroxymethylcytosine levels were observed between fully and partially reprogrammed nuclear transfer embryonic stem cells and induced pluripotent stem cells. Through syngeneic comparison, our study identifies for the first time that Grb10 is associated with the pluripotency state in nuclear transfer embryonic stem cells.

\section{INTRODUCTION}

Reprogramming refers to the erasure and remodeling of epigenetic marks during mammalian development in vivo and is an approach that changes differentiated cells into dedifferentiated cells in vitro. Somatic cell nuclear transfer (SCNT) and transcription factor (TF) mediated reprogramming are two major in vitro reprogramming techniques. 
The studies of mammalian cloning and reprogramming have grown substantially since the first somatic cell cloned sheep, Dolly, was born [1]. The derivation of embryonic stem cells (ESCs) from cloned embryos by SCNT was an important achievement, and nuclear transfer ESCs (ntESCs) can be successfully derived from various adult cell types from mice, rhesus macaques, and humans, among others [2-7]. However, the reprogramming efficiency of SCNT limits the applications of ntESCs, although many solutions have been developed to resolve this issue. The addition of trichostatin A (TSA) and scriptaid (SCR) to the culture medium can improve SCNT efficiency [8-11]. Xistdeficient cumulus cells and Sertoli cells have been shown to robustly improve efficiency for mouse SCNT [12], and $\mathrm{Kdm} 4 \mathrm{~A}$ overexpression increased the blastocyst formation rate of human SCNT embryos [13].

Takahashi and Yamanaka demonstrated that pluripotent stem cells can be obtained from mouse embryonic or adult fibroblasts by introducing four transcription factors, Oct3/4, Sox2, c-Myc, and Klf4, under embryonic stem cell culture conditions [14]. Extensive studies examining TF mediated reprogramming were performed following the discovery that induced pluripotent stem cells (iPSCs) can support the full-term development of tetraploid blastocyst complemented embryos in mice $[15,16]$. Many studies have been performed to improve efficiency. Small molecules such as Vitamin $\mathrm{C}$ have been used to improve efficiency both in mouse and human TF mediated pluripotent reprogramming [17]. A recent study found that combining several small molecules can reprogram mouse somatic cells, increasing reprogramming efficiency to $0.2 \%$ [18]. Moreover, the expression of certain genes can improve the TF mediated reprogramming efficiency. Zscan4 overexpression increased iPSCs efficiency and quality in mice [18], whereas $\mathrm{Nr} 5 \mathrm{a} 2$ can replace Oct4 during reprogramming and improve efficiency in mice [19].

To better understand SCNT and TF mediated reprogramming, the methylation state of imprinted mouse genes, epigenetic memory, somatic mutation and telomeric rejuvenation of ntESCs and iPSCs have been compared [20-23]. The DNA methylation and transcriptome profiles of human ntESCs corresponds closely to in vitro fertilized embryonic stem cells (IVFESCs), whereas iPSCs exhibits differences, retaining residual DNA methylation patterns typical of parental somatic cells [24]. Comparisons of ntESCs and iPSCs can be used to identify high-quality ntESCs or iPSCs for future regenerative medicine applications. Previous studies have shown that activation of the Dlk1-Dio3 imprinted genomic region is required for $\mathrm{TF}$ induced iPSCs to obtain full pluripotency and the expression of the imprinted genes Rian and Gtl2 was higher in fully reprogrammed iPSCs than in partially reprogrammed iPSCs [25, 26]. However, it remains unclear whether the Dlk1-Dio3 region is also associated with ntESCs pluripotency state.
In this study, we first generated exactly syngeneic ntESCs and iPSCs from adipocyte progenitor cells (APCs) isolated from the all-iPSC mice through the primary $\mathrm{TF}$ mediated reprogramming in our previous study [15]. This secondary reprogramming system maintained the same genomic insertion in both ntESCs and iPSCs. By comparing fully and partially reprogrammed ntESCs and iPSCs, we observed that imprinted genes Rian and Gt12 in the Dlk1-Dio3 region related to iPSCs pluripotency state were not correlated with the pluripotency state in ntESCs. A previous study has shown that incomplete somatic cell reprogramming caused abnormally high genomic 5-methylcytosine $(5 \mathrm{mC})$ levels in iPSCs compared to ntESCs and ESCs, suggesting that there might be different $5 \mathrm{mC}$ levels between ntESCs and iPSCs [27]. We did not observe a significant difference in $5 \mathrm{mC}$ or 5-hydroxymethylcytosine $(5 \mathrm{hmC})$ levels between fully and partially reprogrammed ntESCs and iPSCs. Our comparison of fully and partially reprogrammed ntESCs demonstrated that Grb10 was associated with the pluripotency state in ntESCs using high throughput sequencing, which was verified with quantitative reverse-transcription PCR in ntESCs from both APCs and fibroblast cells. By using syngeneic comparison, our study provides valuable information regarding ntESCs and iPSCs and identifies for the first time an important gene associated with the pluripotency state in ntESCs.

\section{RESULTS}

\section{The derivation of ntESCs and iPSCs from APCs in a secondary reprogramming system}

To perform an exact syngeneic comparison of ntESCs and iPSCs in this study, a secondary reprogramming system was established.

APCs isolated from the $1^{0}$-all-iPSC mice were used to derive ntESCs and to generate iPSCs $[15,28-31]$. The $1^{0}$-mouse embryonic fibroblasts (MEFs)-iPSC-37 cells (37iPSC) were derived from 13.5 days postcoitum (dpc) embryos collected from female 129S2/Sv mice mated with Rosa26-M2rtTA transgenic mice and were shown to be fully pluripotency by their capacity to generate all-iPSC mice.

NtESCs were derived from the blastocysts of SCNT embryos. SCNT embryos were obtained by transferring the nuclei of APCs into enucleated oocytes (Table 1). SCNT blastocysts were plated onto a feeder layer of MEFs, and outgrowths emerged after approximately 5 to 10 days. In total, $38 \mathrm{ntESCs}$ cell lines were established from 440 cloned embryos.

IPSCs induced from APCs were generated by adding doxycycline. After approximately 7 days, ESlike colonies emerged. In total, 45 iPSCs cell lines were established. Hereafter, we designate the ntESCs and iPSCs from APCs using different derivation methods as AN and AI, respectively. 
Table 1: Summary of ntESC establishment from cloned embryos with APCs

\begin{tabular}{lcccc}
\hline Type of donor cells & No. cloned embryos & $\begin{array}{c}\text { No. morula/ } \\
\text { blastocysts (\%) }\end{array}$ & No. ICM outgrowths & $\begin{array}{c}\text { No. ntES cell lines } \\
(\%)\end{array}$ \\
\hline APCs & 187 & $7(3.7)$ & 0 & $0(0)$ \\
APCs & 131 & $56(42.7)$ & 19 & $19(14.5)$ \\
APCs & 122 & $54(44.3)$ & 19 & $19(15.6)$ \\
\hline Total & 440 & $117(26.6)$ & 38 & $38(8.6)$ \\
\hline
\end{tabular}

Abbreviation: APCs, adipocyte progenitor cells; ntESCs, nuclear transfer embryonic stem cells.

$\%$ : percentage of reconstructed embryos

\section{Characterization of different pluripotency states in ntESCs and iPSCs derived from APCs}

To evaluate the pluripotency state in syngeneic ntESCs and iPSCs, we examined the karyotypes of the derived cell lines and identified $31 \mathrm{AN}$ cell lines and 41 AI cell lines with normal karyotypes (Table 2). There were only minor differences in the percentage of cell lines with a normal karyotype between the AN and AI cell lines.

Next, we observed that AN and AI cell lines exhibited a typical mouse ESCs morphology, with a compact appearance and a well-defined border. The cell lines were also positive for AP activity (Figure 1A). AN and AI cell lines expressed both protein and mRNA for pluripotency marker genes (Figure 1B and 1C).

Furthermore, we examined the developmental potential of the cell lines using teratoma formation as in vivo differentiation assay. Histological examination (H\&E) revealed that AN and AI could give rise to teratomas containing tissues from all three germ layers (Figure 1D and Supplementary Table 1). In addition, under in vitro differentiation conditions, differentiated cells derived from AN and AI cell lines exhibited upregulated markers for all three germ layers compared to undifferentiated cell lines (Figure 1E and $1 \mathrm{~F}$ ).

To investigate the pluripotency state of $\mathrm{AN}$ and AI cell lines, we utilized a tetraploid complementation assay and performed germline transmission (Figure $1 \mathrm{G}$ and Supplementary Table 1). We identified four fully pluripotent ntESCs (AN1, AN9, AN15 and AN20), five partially reprogrammed ntESCs (AN2, AN3, AN5, AN6 and AN7), three fully pluripotent iPSCs (AI3, AI7 and AI10) and one partially reprogrammed iPSCs (AI9). Hereafter, we designated the fully pluripotent ntESCs as $\mathrm{AN} \mathrm{F}$, the partially reprogrammed ntESCs as AN P, the new derived fully pluripotent iPSCs and previous identified fully pluripotent GS32 as AI F, and the new derived partially reprogrammed AI9 and previous identified partially GS4 and GS9 as AI P, respectively. These fully and partially pluripotent cell lines were utilized for further investigation.

\section{Expression of Rian and Gt12 in the Dlk1-Dio3 region in ntESCs and iPSCs}

Previous studies have shown that the imprinted genes Rian and Gt12, which are located in the Dlk1-Dio3 region, are associated with pluripotency state in mouse iPSCs $[32,33]$. The quantitative PCR results showed that the expression levels of Rian and Gt12 were significantly different between AI F and AI P, which is consistent with previous reports showing that Rian and Gt12 were expressed at a higher level in fully reprogrammed iPSCs than in partially reprogrammed iPSCs $[25,26]$. However, we found that there was no significant difference in the expression of Rian and Gt12 between AN F and AN P (Figure 2A and 2B), suggesting that pluripotency state related genes in the Dlk1-Dio3 region in iPSCs might not be associated with pluripotency in ntESCs. Rtl1 is another gene in the Dlk1-Dio3 region that showed little expression difference between AN F and AN P, but there was a significant difference in Rtll expression between AI $\mathrm{F}$ and AI P (Figure 2C).

\section{5mC and $5 \mathrm{hmC}$ DNA modifications in ntESCs and iPSCs were not different}

Evaluation of the epigenetic modification in ESCs, ntESCs and iPSCs suggested that incomplete somatic cell reprogramming might be caused by abnormally high levels of genomic $5 \mathrm{mC}$ in iPSC lines, even though iPSCs had germ-line chimeric properties [27]. Here, we examined the $5 \mathrm{mC}$ and $5 \mathrm{hmC}$ levels in ntESCs and iPSCs and determined whether $5 \mathrm{mC}$ content correlated with fully or partially reprogrammed stem cells. Our results showed that there was no significant difference in $5 \mathrm{mC}$ or $5 \mathrm{hmC}$ levels in AN F and AN P cell lines, and AI F and AI P cell lines (Figure 3A and Figure 3B).

\section{The identification of Grb10 associated with pluripotency state in ntESCs}

We used high throughput sequencing to compare gene expression differences between AN F and AN P, and AI F and AI P. In AN F, 84 genes were significantly upregulated, 
Table 2: Summary of karyotype analysis in ntESCs and iPSCs

\begin{tabular}{lcc}
\hline Type of cell lines & No. cell lines & No. normal karyotype cell lines (\%) \\
\hline AN & 38 & $31(81.6)$ \\
AI & 45 & $41(91.1)$ \\
\hline
\end{tabular}

Abbreviation: AN, nuclear transfer embryonic stem cells and adipocyte progenitor cells as donor cells; AI, induced pluripotent stem cells and adipocyte progenitor cells as primary cells.

$\%$ : percentage of normal karyotype cell lines.

A

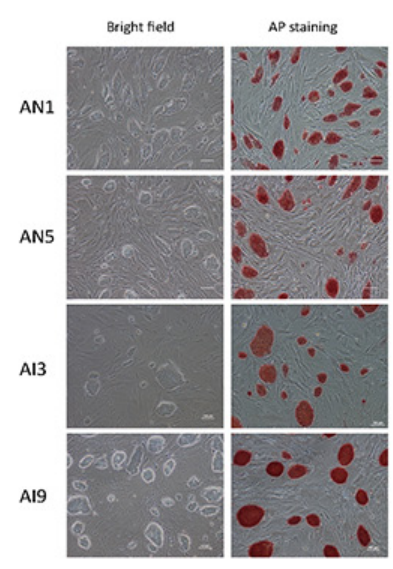

C

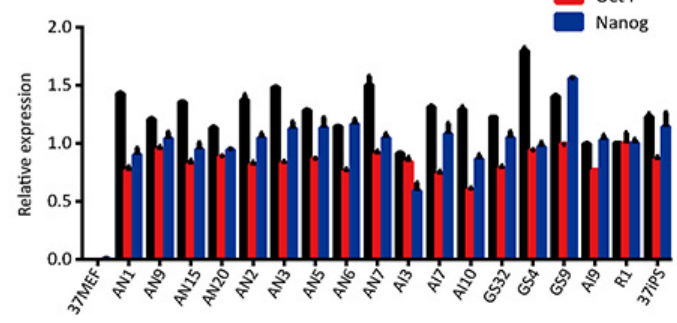

E

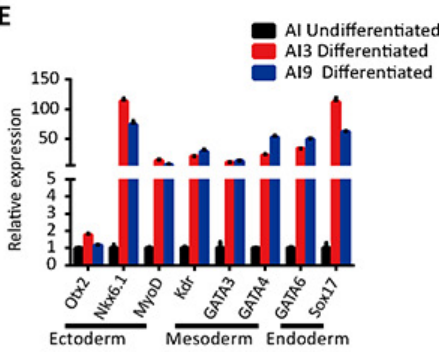

B

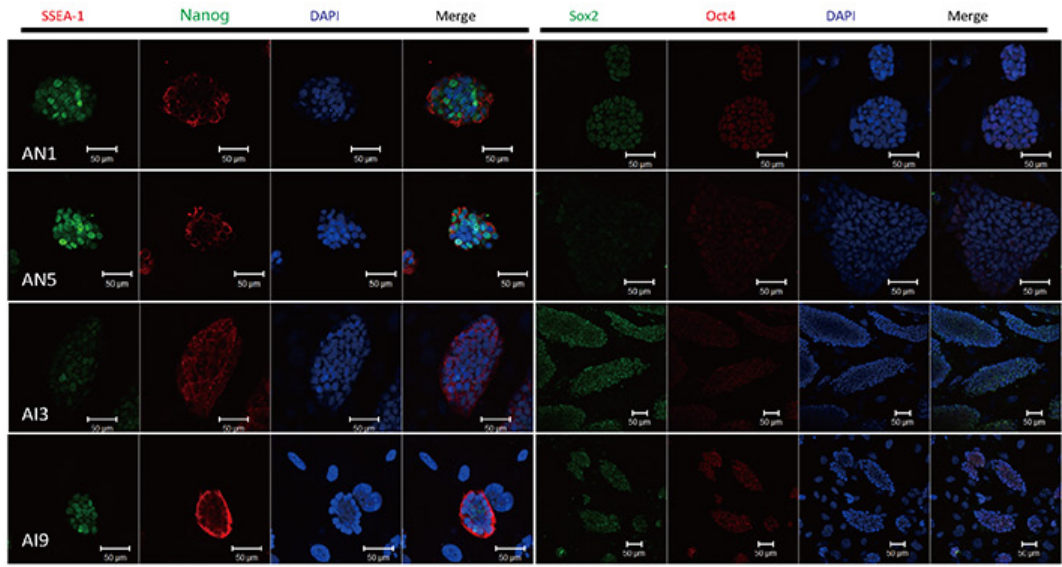

D

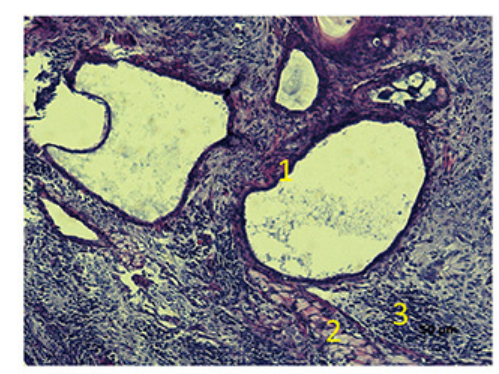

G
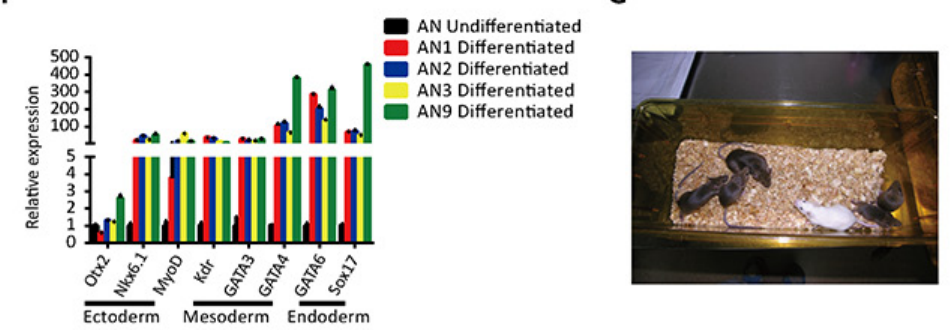

Figure 1: Characteristics of ntESCs and iPSCs from APCs. (A) Morphology and alkaline phosphatase expression of ntESCs and iPSCs. Scale bars, $100 \mu \mathrm{m}$. (B) Immunofluorescence staining of pluripotent markers SSEA-1 (red), Nanog (green), Sox2 (green), and Oct4 (red) in AN and AI lines. Nuclei were stained with DAPI. Scale bars, 50 $\mu \mathrm{m}$. (C) Quantitative PCR analysis of the pluripotent markers Sall4, Oct4 and Nanog in AN and AI lines. Relative mRNA expression is normalized to GAPDH and is represented relative to expression in fibroblasts (37MEF). The experiments were performed in triplicate (means $\pm \mathrm{SD} ; \mathrm{n}=3$ ). The primers used are listed in Supplementary Table 2. (D) H\&E staining of teratomas generated from AN1 that formed tissues from all three germ layers. 1: Ectoderm; 2: Mesoderm; 3: Endoderm. Scale bars, $100 \mu \mathrm{m}$. A detailed description of all of the cell lines is given in Supplementary Table 1. (E and F) Quantitative PCR analysis showing the upregulation of markers for all three germ layers during the in vitro differentiation of AN and AI lines. Relative mRNA expression is normalized to GAPDH and represented relative to expression in undifferentiated AN and AI lines. The experiments were performed in triplicate (mean $\pm \mathrm{SD} ; \mathrm{n}=3$ ). The primers used are listed in Supplementary Table 2. (G) Germline transmission of an AN1 tetraploid mouse. Details on all of the cell lines are in Supplementary Table 1. 




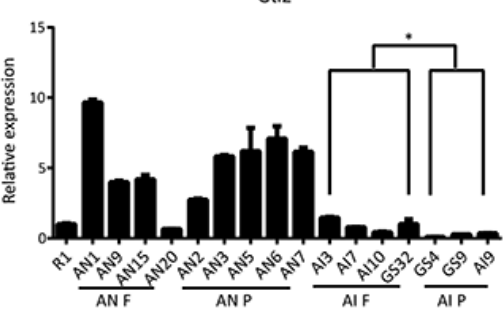



Figure 2: Expression of Rain, Gtl2 and Rtl1 in AN and AI cell lines. (A, B, and C) Quantitative PCR analysis for Rain, Gt12 and Rtl1, which are in the Dlk1-Dio3 region, in AN and AI lines. AN F: full reprogrammed cells as shown by tetraploid complementation assay in AN lines; AN P: partially reprogrammed cells as shown by tetraploid complementation assay in AN lines; AI F: full reprogrammed cells as shown by tetraploid complementation assay in AI lines; AI P: partially reprogrammed cells as shown by tetraploid complementation assay in AI lines. Relative mRNA expression is normalized to GAPDH and is represented relative to expression in R1 ESCs. The experiments were performed in triplicate (means $\pm \mathrm{SD}$; $\mathrm{n}=3$; Student's $\mathrm{t}$ test; ${ }^{*} \mathrm{p}<0.05$; ${ }^{*} \mathrm{p}<0.01$, and ${ }^{* * *} \mathrm{p}<0.001$ ). The primers used are listed in Supplementary Table 2 .

A

$5 \mathrm{mC}$



B

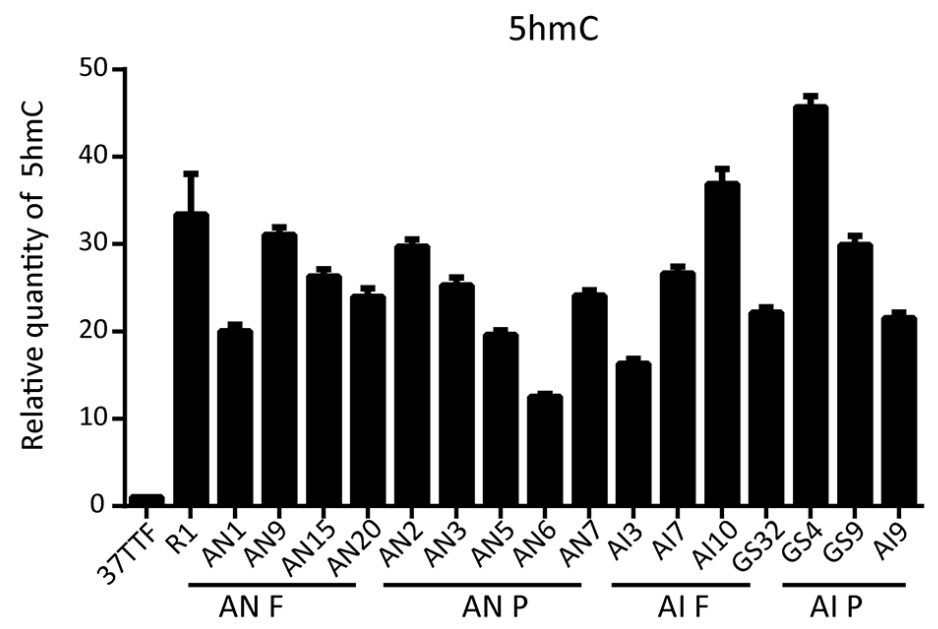

Figure 3: Quantification of $5 \mathrm{mC}$ and $5 \mathrm{hmC}$ in $\mathrm{AN}$ and $\mathrm{AI}$ lines. (A) Quantification of genomic $5 \mathrm{mC}$ in AN F, AN P, AI F and AI P cell lines showing values that were normalized and compared to fibroblasts (37 TTF). Experiments were performed in triplicate (mean \pm SD; $\mathrm{n}=3$ ). (B) Quantification of genomic $5 \mathrm{hmC}$ in AN F, AN P, AI F and AI P cell lines showing values that were normalized and compared to fibroblasts (37TTF). Experiments were performed in triplicate (mean $\pm \mathrm{SD} ; \mathrm{n}=3$ ). 
and 391 genes were upregulated in AI F compared to AN $\mathrm{P}$ and AI P, respectively. Gene Ontology (GO) analysis showed that most of the upregulated genes in AN F were related to transcription (Figure 4A). The upregulated genes in AI F were enriched for mesodermal developmentrelated processes including blood vessel, vasculature and skeletal development (Figure 4B). Interestingly, we found that Grb10, a maternally imprinted gene, was upregulated in both the AI F and AN F cell lines (Figure 4C). Our quantitative PCR analysis confirmed that Grb10 expression in fully reprogrammed AN cells was higher than in partially reprogrammed cells (Figure 4D). In AI cell lines, Grb10 expression in most AI fully reprogrammed cell lines was higher than in partially reprogrammed cell lines except for AI9 (Figure 4E). These results suggest that Grb10 might be associated with pluripotency state in ntESCs. To further verify that Grb10 was associated with pluripotency state in
ntESCs, ntESCs cells (FN) derived from SCNT blastocysts using the tail-tip fibroblasts (TTFs) as donor cells were examined. The TTFs were from the $1^{0}$-all-iPSC mice in the primary $\mathrm{TF}$ induced pluripotent reprogramming in our previous study [15]. The $1^{0}$-MEF-iPSC-37 cells (37iPSC) were derived from 13.5 dpc embryos collected from female $129 \mathrm{~S} 2 / \mathrm{Sv}$ mice mated with Rosa26-M2rtTA transgenic mice and were shown to be fully pluripotency by their capacity to generate the all-iPSC mice. FN cell lines were also grouped into fully and partially reprogrammed cell lines using the tetraploid complementation assay and were designated FN F and FN P, respectively (unpublished data). This assay showed that Grb10 expression was significantly higher in FN F than in FN P (Figure 4F), which indicates that Grb10 might work as an important molecular marker for indicating pluripotency state in ntESCs derived from different cell types.
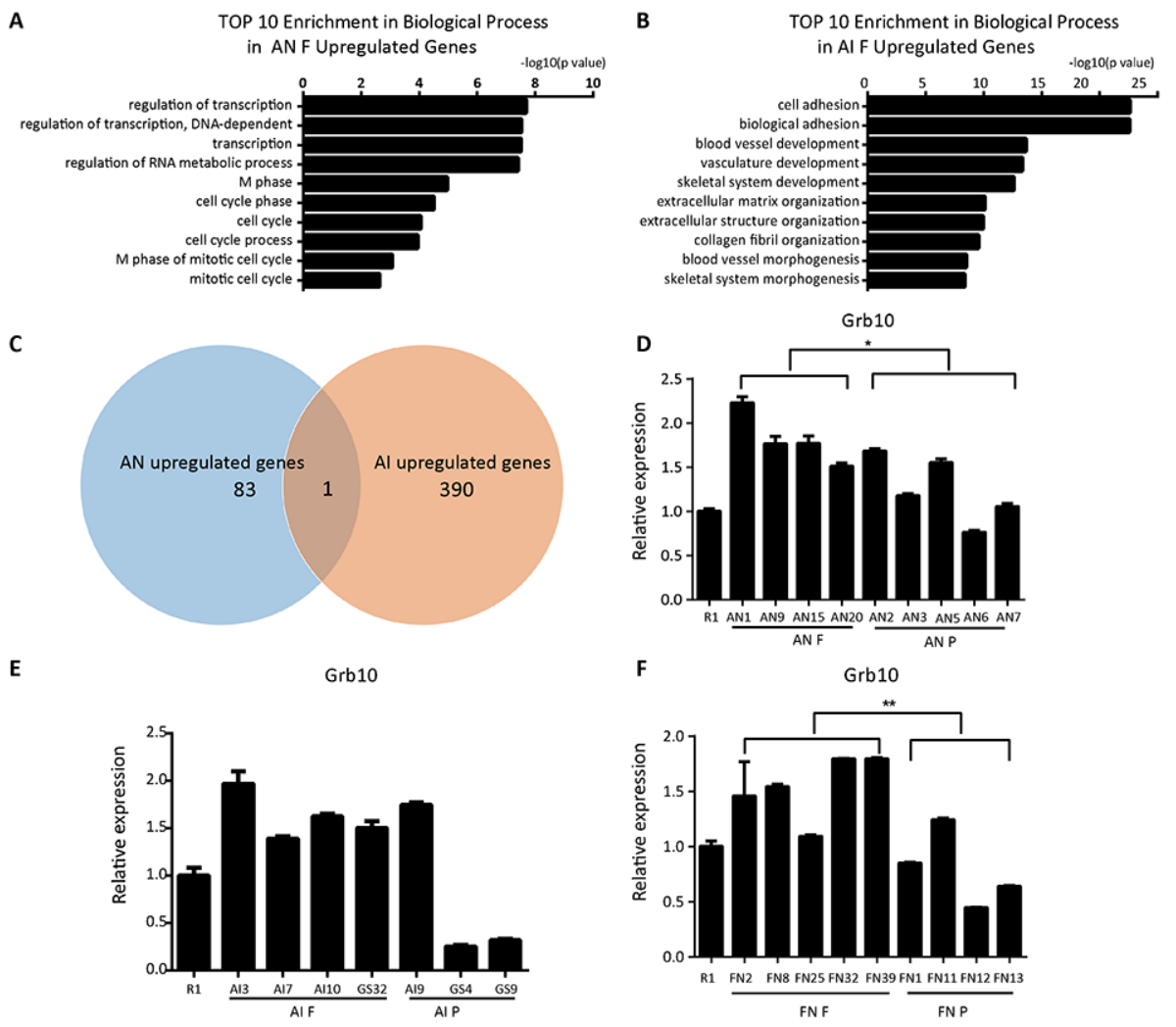

Figure 4: Grb10 is associated with the pluripotency state in ntESCs. (A) GO analysis based on the upregulated genes from the comparison of fully and partially reprogrammed AN cells performed by David Bioinformatics Resources. (B) GO analysis based on the upregulated genes from the comparison of fully and partially reprogrammed AI cells performed by David Bioinformatics Resources. (C) Venn diagram representing the overlap between AI upregulated genes and AN upregulated genes. The sizes of the overlapping areas are not proportional to the actual number of genes. (D, E and F) Quantitative PCR analysis of Grb10 in AN, AI and FN cell lines. AN F: fully reprogrammed cells by tetraploid complementation assay in AN cell lines; AN P: partially reprogrammed cells by tetraploid complementation assay in AN cell lines; AI F: fully reprogrammed cells by tetraploid complementation assay in AI cell lines; AI P: partially reprogrammed cells by tetraploid complementation assay in AI cell lines; FN F: fully reprogrammed cells by tetraploid complementation assay in FN cell lines; FN P: partially reprogrammed cells by tetraploid complementation assay in FN cell lines. Relative mRNA expression is normalized to GAPDH and is represented relative to expression in R1 ESCs. The experiments were performed in triplicate (means \pm SD; $\mathrm{n}=3$; Student's t test; ${ }^{*} \mathrm{p}<0.05$; $* \mathrm{p}<0.01$, and $\left.* * * \mathrm{p}<0.001\right)$. And there is no significant difference in the inside of the AIF, AIP, ANF and ANP groups ( $n=3, F>0.05$, one-way ANOVA). The primers used are listed in Supplementary Table 2. 


\section{DISCUSSION}

In this study, we compared syngeneic ntESCs and iPSCs and showed that the expression of pluripotency associated genes in Dlk1-Dio3 region and $5 \mathrm{mC} / 5 \mathrm{hmC}$ levels could not be used to evaluate fully and partial reprogrammed ntESCs, and demonstrated that Grb10 is associated with the pluripotency state in ntESCs.

Imprinted genes are expressed from a single parental allele and have parental-specific epigenetic modifications [34]. The imprinted genes Rian and Gt12 are located in Dlk1-Dio3 region on distal mouse chromosome 12 [32, 33]. A previous study indicated that Rian and Gt12 are significantly downregulated transcripts in iPSCs when comparing genome wide expression between genetically identical mouse ESCs and iPSCs [25]. In our study, the expression of Rian, Gt12 and Rtl1 was higher in AI F than in AI P and was associated with iPSCs quality, which was consistent with the previous studies [25, 26]. It remains unclear that whether the expression of Rian, Gt12 and Rt11 are also associated with the pluripotency state in ntESCs. To evaluate the pluripotency state in ntESCs, we examined these associated genes in more AN cell lines in this study. There was little difference in the expression of Rian, Gt12, and Rtl1 between AN F and AN P, which indicated that Rian, Gt12 and Rtl1 expression from the Dlk1-Dio3 region is not useful to evaluate fully and partial reprogrammed ntESCs. A previous study indicated that TF stoichiometry influences the Dlk1-Dio3 locus and that some Gt12LOW (not OFF) iPSCs can support the iPSC full-term development [35]. Gt12 might activate the Dlk1-Dio3 region and be used to assess the quality of reprogramming in iPSCs [36]. It showed that the expression of Gt12 was associated to the quality of iPSCs. Our results showed that Gt12 expression in AN cell lines was not associated with the pluripotency state in ntESCs and Gtl2 could not be used to assess the quality of reprogramming in ntESCs.

Epigenetic modifications include DNA methylation and histone modification, which play important roles in both SCNT reprogramming and TF induced pluripotent reprogramming. Cloning of mammals by SCNT results in gestational or neonatal failure with at most a few percent of manipulated embryos resulting in live births likely due to the inappropriate epigenetic reprogramming [35]. The identity of somatic cells is strictly protected by an epigenetic barrier, and these cells acquire pluripotency by breaking the epigenetic barrier by reprogramming factors such as Oct3/4, Sox2, Klf4, Myc and LIN28 [37, 38]. A previous report indicates that a major reprogramming event during early embryonic development is the erasure and subsequent re-establishment of methylation patterns at $5 \mathrm{mC}$ [39]. $5 \mathrm{hmC}$ is an epigenetic modification that has been suggested to be associated with the pluripotency state during reprogramming of mouse fibroblasts into iPSCs [40]. Our previous results suggested that $5 \mathrm{mC}$-to- $5 \mathrm{hmC}$ conversion represents a crucial step in the initiation of epigenetic remodeling and transcriptome resetting to achieve pluripotency [41]. A previous report found that the genomic $5 \mathrm{mC}$ levels in iPSCs were higher than the levels in ntESCs [27]. Therefore, we compared the levels of $5 \mathrm{mC}$ and $5 \mathrm{hmC}$ in both ntESCs and iPSCs. However, we found no difference in $5 \mathrm{mC}$ and $5 \mathrm{hmC}$ levels between the AN F and AN P cell lines, or the AI F and AI P cell lines. This result was not consistent with previous study that reported ntESCs and iPSCs have different $5 \mathrm{mC}$ levels. In this study, we used nine ntESC cell lines and seven iPSC cell lines, which were syngeneic with same genomic insertion, to examine $5 \mathrm{mC}$ and $5 \mathrm{hmC}$ levels. These stem cells were well defined as fully and partially reprogrammed ntESCs and iPSCs by tetraploid complementation assay. We used more cell lines than the previous study, which used only two ntESCs and two germ-line chimeric iPSCs without further performing tetraploid complementation assay. Our results suggest that genomic $5 \mathrm{mC}$ levels might not be used to evaluate pluripotency state in AN and AI cell lines and that the dynamic conversion of $5 \mathrm{mC}$ to $5 \mathrm{hmC}$ might become stable after reprogramming is complete.

To evaluate the pluripotency state of AN cell lines, a comparison of fully and partially reprogrammed cell lines was performed using high throughput sequencing. To avoid the difference caused by RNA library construction, quality control, quantification and other sequencing processes, we performed high throughput sequencing in all AN and AI cell lines together, including previous identified AI cell lines. Interestingly, our result showed a significant difference between AN F and AN P in the expression levels of Grb10 (Figure 4D), which suggested that Grb10 might be a marker for the pluripotency state in ntESCs. Grb10, which is also called Meg1, is an imprinted gene on mouse proximal chromosome 11 and a candidate gene causing Silver-Russell syndrome [42]. Previous reports suggested that aberrant function of Grb10 may contribute to disorders of proliferation, apoptosis, and metabolism, with specific emphasis on growth and neuronal development [43-46]. Recent study has indicated that Grb10 plays an inhibitory role for hematopoietic stem cell self-renewal and regeneration [47]. We examined Grb10 expression in another series of fully and partially reprogrammed ntESCs derived from fibroblasts and verified that Grb10 is associated with the pluripotency state in ntESCs in our study, although the mechanism for this association remains unclear.

In summary, our study performed an invaluable comparison of syngeneic ntESCs and iPSCs and identified for the first time that an imprinted gene, Grb10, is associated with the pluripotency state in ntESCs.

\section{MATERIALS AND METHODS}

\section{Mice and cell culture}

All of the animal protocols and experiments were approved by the Animal Research Committee of the 
Institute of Zoology, Chinese Academy of Sciences, and are consistent with the National Institute of Biological Sciences guide for the care and use of laboratory animals.

Female B6D2F1 (C57BL/6XDBA/2) mice (8-10 weeks old) were superovulated by sequential injection with 7 IU of pregnant mare serum gonadotropin (PMSG) and human chorionic gonadotropin (hCG) (San-Sheng Pharmaceutical Co. Ltd, Ningbo, China). Metaphase II (MII) oocytes were collected for SCNT experiments as previously described [48].

MEFs were derived from 13.5 dpc embryos collected from ICR mice and were cultured in Fundamental Culture medium (FM) containing Dulbecco's modified Eagle's medium (DMEM) (Invitrogen, Thermo Fisher Scientific, Waltham, MA, USA) supplemented with $10 \%(\mathrm{v} / \mathrm{v})$ fetal bovine serum (FBS) (Invitrogen, Thermo Fisher Scientific) and $1 \mathrm{mM}$ L-glutamine (Merck, Millipore, Billerica, MA, USA). ESCs culture medium contains DMEM (Merck, Millipore) supplemented with 15\% (v/v) FBS (Hyclone, Logan, Utah), 1 mM L-glutamine (Merck, Millipore), $0.1 \mathrm{mM}$ mercaptoethanol (Merck, Millipore), 1\% nonessential amino acid stock (Merck, Millipore), penicillin/ streptomycin (Merck, Millipore), nucleosides (Merck, Millipore), and 1,000 U/ml Leukemia Inhibitory Factor (LIF) (Merck, Millipore).

Adipocyte progenitor cells $\left(1^{0}-\mathrm{APCs}\right), 37$ tailtip fibroblasts $\left(1^{0}\right.$-TTFs $)$ and $37 \mathrm{MEFs}\left(1^{0}\right.$-MEFs $)$ were isolated from $1^{0}$-all-iPSC mice produced by $1^{0}$-MEFiPSC-37 cells, which were shown to be fully pluripotent by their capacity to generate all-iPSC mice [15].

AN cell lines were derived from SCNT blastocysts using $1^{0}$-APCs as donor cells. AI cell lines were generated from $1^{0}$-APCs by adding doxycycline to the induction medium. Previously well-defined $2^{0}$-APC-iPSC-4, $2^{0}$-APC-iPSC-9, and $2^{0}$-APC-iPSC-32 cell lines were named GS4, GS9, and GS32, respectively, for these experiments [28-31].

FN cell lines were derived from SCNT blastocysts using $1^{0}$-TTFs as the donor cells.

A schematic of the cell lines derivation is shown in Supplementary Figure 1.

\section{Alkaline phosphatase (AP) staining}

AP staining was performed with a Leukocyte Alkaline Phosphatase Kit (Sigma, St Louis, MO, USA) according to the manufacturer's instructions.

\section{Karyotype analysis}

The cells were incubated in ESCs medium with 0.25 $\mu \mathrm{g} / \mathrm{ml}$ colcemid (Invitrogen, Thermo Fisher Scientific) for 2-3 $\mathrm{h}$ and harvested with $0.05 \%$ Trypsin-EDTA (Invitrogen, Thermo Fisher Scientific). After incubation in hypotonic solution containing $0.4 \%$ sodium citrate and $0.4 \%$ potassium chloride $(1: 1, \mathrm{v} / \mathrm{v})$ at $37^{\circ} \mathrm{C}$ for $5 \mathrm{~min}$, the cells were fixed with a methanol/acetic acid mixture (3:1, $\mathrm{v} / \mathrm{v})$. The fixed cells were mounted on glass slides and stained with Giemsa at $37^{\circ} \mathrm{C}$ for $10-15$ min after drying. At least 20 metaphase chromosome karyoschisis were examined for each cell line.

\section{Immunofluorescence staining}

For immunofluorescence staining, AN and AI cells were seeded on gelatin-coated cover slips and fixed with 4\% paraformaldehyde. After permeabilization with $0.5 \%$ Triton-X and blocking with $0.5 \%$ bovine serum albumin (BSA), the cells were incubated with primary antibodies against Oct4 (1:500, Santa Cruz, Dallas, TX, USA), Sox2 (1:500, Santa Cruz), Nanog (1:500, COSMO BioCo, Tokyo, Japan) and SSEA-1 (1:50, Merck, Millipore). Then, the cells were incubated with the appropriate secondary antibodies after washing three times. DNA was labeled with DAPI (Merck, Millipore). Stained cells were mounted on cover slips and observed using a LSM 510 META microscope (Zeiss, North York, ON, Canada).

\section{Teratoma formation}

AN and AI cells $\left(2-5 \times 10^{6}\right)$ were subcutaneously injected into the groin of severe combined immune deficiency (SCID) mice. Tumors were dissected and processed for hematoxylin-eosin staining 6-8 weeks after injection.

\section{Embryoid body formation}

AN and AI cells were trypsinized into a single cell suspension and transferred to Petri dishes in DMEM supplemented with $15 \%$ FBS without LIF. Three to seven days later, the embryoid bodies (EBs) were harvested and plated onto gelatin-coated tissue culture dishes for another 3-7 days. Total RNA from plated EBs was extracted and used for quantitative PCR. GAPDH was used as an endogenous control.

\section{Quantitative reverse-transcription PCR}

Total RNA was purified using TRIzol (Invitrogen, Thermo Fisher Scientific). RNA $(2 \mu \mathrm{g})$ was reversetranscribed using M-MLV Reverse Transcriptase and RNasin RNase Inhibitor (Promega, Madison, WI, USA). Quantitative reverse-transcription PCR was performed using SYBR Premix Ex Taq (Takara, Kusatsu, Japan). The reactions were performed in triplicate on a $1 / 10$ dilution of the cDNA obtained from above. Gene expression in each sample was normalized to GAPDH, and the relative quantification of expression was estimated using the comparative CT method. All of the primers used are listed in Supplementary Table 2. 


\section{Tetraploid complementation}

To perform tetraploid complementation, B6D2F1 embryos at the 2-cell stage were electrofused to tetraploid embryos, 10-15 cells were injected into the reconstructed tetraploid blastocysts, and these were transplanted into the uteri of pseudo-pregnant mice. Caesarean sections were performed on day 19.5, and pups were fostered by lactating ICR mothers.

\section{RNA-seq data analysis}

Total RNA was extracted from the different cell lines using TRIzol (Invitrogen, Thermo Fisher Scientific). Libraries were constructed with the NEBNext DNA Library Prep Master Mix Set for Illumina (New England Biolabs, MA, USA), and PCR products were purified using AMPure XP beads (Beckman, Brea, CA, USA). The RNA library was quantified using Qubit 1.0 (Invitrogen, Thermo Fisher Scientific), analyzed using an Agilent 2100 Bioanalyzer (Agilent Technologies, Santa Clara, CA, USA) for size distribution, and then sequenced with an Illumina Hiseq-2500 in single mode (1x50nt) by the Bioinformatics core facility at National institute of Biological Sciences, Beijing.

The $51 \mathrm{bp}$ sequences called by the Illumina pipeline were mapped to the mouse genome ( $\mathrm{mm} 9)$ using Tophat (v2.1.0) for data analysis. Gene annotation and calculation of FPKM values was performed using Cufflinks (v2.2.1) with the GTF annotation file (mm9).

Gene expression differences were assessed by Cuffdiff with a false discovery rate correction for multiple testing. Genes with a p-value $<0.05$ and q-value $<0.05$ were considered differentially expressed.

To identify overrepresented biological categories within each cluster, GO analysis was applied (DAVID, https://david.ncifcrf.gov/).

\section{Liquid chromatography-mass spectrometry (LC- MS/MS) Analysis of $5 \mathrm{mC}$ and $5 \mathrm{hmC}$}

The genomic DNA (5 $\mu \mathrm{g}$ ) from different cell lines was analyzed by liquid chromatography-tandem mass spectrometry to assess the quantity of $5 \mathrm{mC}$ and $5 \mathrm{hmC}$, as described in our previous study $[41,49,50]$.

\section{Accession numbers}

All the high-throughput sequencing data sets can be accessed as the GEO reference GSE92308 (https://www. ncbi.nlm.nih.gov/geo/query/acc.cgi?token=ezmjmmkmpf cxngv\&acc $=$ GSE92308).

\section{Statistics}

Student's t tests were performed using SigmaStat 3.5 software for statistical comparisons. And one-way
ANOVA were performed using SPSS Statistics 19 software.

\section{Abbreviations}

5hmC, 5-hydroxymethylcytosine;

$5 \mathrm{mC}, 5$-methylcytosine;

AI F, fully reprogrammed cells by tetraploid complementation assay in AI cell lines;

AI P, partially reprogrammed cells by tetraploid complementation assay in AI cell lines;

$\mathrm{AI}$, induced pluripotent stem cells and adipocyte progenitor cells as primary cells;

AN F, fully reprogrammed cells by tetraploid complementation assay in AN cell lines;

AN P, partially reprogrammed cells by tetraploid complementation assay in AN cell lines;

AN, nuclear transfer embryonic stem cells and adipocyte progenitor cells as donor cells;

AP Staining, Alkaline Phosphatase staining;

$\mathrm{APCs} / 1^{0}$-APCs, adipocyte progenitor cells;

B6D2F1 mice, C57BL/6XDBA/2 mice;

BSA, bovine serum albumin;

DMEM, Dulbecco's modified Eagle's medium;

dpc, days postcoitum;

EBs, Embryoid bodies;

ESC, embryonic stem cell

FBS, fetal bovine serum;

FM, Fundamental Culture medium;

FN F, fully reprogrammed cells by tetraploid complementation assay in FN cell lines;

FN P, partially reprogrammed cells by tetraploid complementation assay in FN cell lines;

FN, nuclear transfer embryonic stem cells and tailtip fibroblasts as donor cells;

GO analysis, Gene Ontology analysis;

GS32, 20-APC-iPSC-32 cell line;

GS4, $2^{0}$-APC-iPSC-4 cell line;

GS9, $2^{0}$-APC-iPSC-9 cell line;

H\&E, Histological examination;

hCG, human chorionic gonadotropin;

iPSCs, induced pluripotent stem cells;

IVF-ESCs, in vitro fertilized embryonic stem cells;

LC-MS/MS, Liquid Chromatography-Mass Spectrometry.

LIF, Leukemia Inhibitory Factor;

MEFs, mouse embryonic fibroblasts;

MII, Metaphase II;

ntESCs, nuclear transfer embryonic stem cells

PMSG, pregnant mare serum gonadotropin;

SCID mice, severe combined immune deficiency mice;

SCNT, somatic cell nuclear transfer;

SCR, scriptaid;

$\mathrm{TF}$, transcription factor;

TSA, trichostatin A;

TTFs $/ 1^{0}$-TTFs, tail-tip fibroblasts; 


\section{Authors' contributions}

H. Li: conception and design, collection and assembly of the data, data analysis and interpretation, and manuscript writing. S. Gao, H. Huang, W. Liu, X. Liu, Y. Gao, R. Le, X. Kou, Y. Zhao, Z. Kou, J. Li, H. Wang, Y. Zhang, H. Wang: provision of study material and collection and/or assembly of the data. H. Huang and T. Cai: provision of computational analysis. Q. Sun: provision of study material. S. Gao and Z. Han: conception and design, data analysis and interpretation, manuscript writing, and final approval of the manuscript.

\section{ACKNOWLEDGMENTS}

We are grateful to our colleagues Yuanfan Chen, Chenchen Wang, Wei Liu, Yonghua Jiang, Yueying Li and Liang Ran etc. in the laboratories for their assistance with the experiments.

\section{CONFLICTS OF INTEREST}

The authors indicate no potential conflicts of interest.

\section{FUNDING}

This work was supported by This work was supported by Ministry of Science and Technology of China (Grant No. 2014CB964803) and National Natural Science Foundation of China (Grant No. 31325019, 31372144 and 31572226).

\section{REFERENCES}

1. Wilmut I, Schnieke AE, McWhir J, Kind AJ, Campbell KH. Viable offspring derived from fetal and adult mammalian cells. Nature. 1997; 385: 810-3.

2. Kawase E, Yamazaki Y, Yagi T, Yanagimachi R, Pedersen RA. Mouse embryonic stem (ES) cell lines established from neuronal cell-derived cloned blastocysts. Genesis. 2000; 28: 156-63.

3. Munsie MJ, Michalska AE, O'Brien CM, Trounson AO, Pera MF, Mountford PS. Isolation of pluripotent embryonic stem cells from reprogrammed adult mouse somatic cell nuclei. Curr Biol. 2000; 10: 989-92.

4. Wakayama S, Mizutani E, Kishigami S, Thuan NV, Ohta H, Hikichi T, Bui HT, Miyake M, Wakayama T. Mice cloned by nuclear transfer from somatic and ntES cells derived from the same individuals. J Reprod Dev. 2005; 51: 765-72.

5. Byrne JA, Pedersen DA, Clepper LL, Nelson M, Sanger WG, Gokhale S, Wolf DP, Mitalipov SM. Producing primate embryonic stem cells by somatic cell nuclear transfer. Nature. 2007; 450: 497-502.
6. Sparman M, Dighe V, Sritanaudomchai H, Ma H, Ramsey C, Pedersen D, Clepper L, Nighot P, Wolf D, Hennebold J, Mitalipov S. Epigenetic reprogramming by somatic cell nuclear transfer in primates. Stem Cells. 2009; 27: 1255-64.

7. Tachibana M, Amato P, Sparman M, Gutierrez NM, Tippner-Hedges R, Ma H, Kang E, Fulati A, Lee HS, Sritanaudomchai H, Masterson K, Larson J, Eaton D, et al. Human embryonic stem cells derived by somatic cell nuclear transfer. Cell. 2013; 153: 1228-38.

8. Kishigami S, Mizutani E, Ohta H, Hikichi T, Thuan NV, Wakayama S, Bui HT, Wakayama T. Significant improvement of mouse cloning technique by treatment with trichostatin A after somatic nuclear transfer. Biochem Biophys Res Commun. 2006; 340: 183-9.

9. Kishigami S, Bui HT, Wakayama S, Tokunaga K, Van Thuan N, Hikichi T, Mizutani E, Ohta H, Suetsugu R, Sata T, Wakayama T. Successful mouse cloning of an outbred strain by trichostatin A treatment after somatic nuclear transfer. J Reprod Dev. 2007; 53: 165-70.

10. Inoue K, Oikawa M, Kamimura S, Ogonuki N, Nakamura T, Nakano T, Abe K, Ogura A. Trichostatin A specifically improves the aberrant expression of transcription factor genes in embryos produced by somatic cell nuclear transfer. Sci Rep. 2015; 5: 10127.

11. Van Thuan N, Bui HT, Kim JH, Hikichi T, Wakayama S, Kishigami S, Mizutani E, Wakayama T. The histone deacetylase inhibitor scriptaid enhances nascent mRNA production and rescues full-term development in cloned inbred mice. Reproduction. 2009; 138: 309-17.

12. Inoue K, Kohda T, Sugimoto M, Sado T, Ogonuki N, Matoba S, Shiura H, Ikeda R, Mochida K, Fujii T, Sawai K, Otte AP, Tian XC, et al. Impeding Xist expression from the active $\mathrm{X}$ chromosome improves mouse somatic cell nuclear transfer. Science. 2010; 330: 496-9.

13. Chung YG, Matoba S, Liu Y, Eum JH, Lu F, Jiang W, Lee JE, Sepilian V, Cha KY, Lee DR, Zhang Y. Histone Demethylase Expression Enhances Human Somatic Cell Nuclear Transfer Efficiency and Promotes Derivation of Pluripotent Stem Cells. Cell Stem Cell. 2015; 17: 758-66.

14. Takahashi K, Yamanaka S. Induction of pluripotent stem cells from mouse embryonic and adult fibroblast cultures by defined factors. Cell. 2006; 126: 663-76.

15. Kang L, Wang J, Zhang Y, Kou Z, Gao S. iPS cells can support full-term development of tetraploid blastocystcomplemented embryos. Cell Stem Cell. 2009; 5: 135-8.

16. Zhao XY, Li W, Lv Z, Liu L, Tong M, Hai T, Hao J, Guo CL, Ma QW, Wang L, Zeng F, Zhou Q. iPS cells produce viable mice through tetraploid complementation. Nature. 2009; 461: 86-90.

17. Esteban MA, Wang T, Qin B, Yang J, Qin D, Cai J, Li W, Weng Z, Chen J, Ni S, Chen K, Li Y, Liu X, et al. Vitamin $\mathrm{C}$ enhances the generation of mouse and human induced pluripotent stem cells. Cell Stem Cell. 2010; 6: 71-9. 
18. Jiang J, Lv W, Ye X, Wang L, Zhang M, Yang H, Okuka M, Zhou C, Zhang X, Liu L, Li J. Zscan4 promotes genomic stability during reprogramming and dramatically improves the quality of iPS cells as demonstrated by tetraploid complementation. Cell Res. 2013; 23: 92-106.

19. Heng JC, Feng B, Han J, Jiang J, Kraus P, Ng JH, Orlov YL, Huss M, Yang L, Lufkin T, Lim B, Ng HH. The nuclear receptor $\mathrm{Nr} 5 \mathrm{a} 2$ can replace Oct4 in the reprogramming of murine somatic cells to pluripotent cells. Cell Stem Cell. 2010; 6: 167-74.

20. Chang G, Liu S, Wang F, Zhang Y, Kou Z, Chen D, Gao S. Differential methylation status of imprinted genes in nuclear transfer derived ES (NT-ES) cells. Genomics. 2009; 93: 112-9.

21. Kim K, Doi A, Wen B, Ng K, Zhao R, Cahan P, Kim J, Aryee MJ, Ji H, Ehrlich LI, Yabuuchi A, Takeuchi A, Cunniff KC, et al. Epigenetic memory in induced pluripotent stem cells. Nature. 2010; 467: 285-90.

22. Li Z, Lu H, Yang W, Yong J, Zhang ZN, Zhang K, Deng $\mathrm{H}, \mathrm{Xu}$ Y. Mouse SCNT ESCs have lower somatic mutation load than syngeneic iPSCs. Stem Cell Reports. 2014; 2: 399-405.

23. Le R, Kou Z, Jiang Y, Li M, Huang B, Liu W, Li H, Kou X, He W, Rudolph KL, Ju Z, Gao S. Enhanced telomere rejuvenation in pluripotent cells reprogrammed via nuclear transfer relative to induced pluripotent stem cells. Cell Stem Cell. 2014; 14: 27-39.

24. Ma H, Morey R, O'Neil RC, He Y, Daughtry B, Schultz MD, Hariharan M, Nery JR, Castanon R, Sabatini K, Thiagarajan $\mathrm{RD}$, Tachibana M, Kang E, et al. Abnormalities in human pluripotent cells due to reprogramming mechanisms. Nature. 2014; 511: 177-83.

25. Stadtfeld M, Apostolou E, Akutsu H, Fukuda A, Follett P, Natesan S, Kono T, Shioda T, Hochedlinger K. Aberrant silencing of imprinted genes on chromosome $12 \mathrm{qF} 1$ in mouse induced pluripotent stem cells. Nature. 2010; 465: 175-81.

26. Li W, Zhao XY, Wan HF, Zhang Y, Liu L, Lv Z, Wang XJ, Wang L, Zhou Q. iPS cells generated without c-Myc have active Dlk1-Dio3 region and are capable of producing fullterm mice through tetraploid complementation. Cell Res. 2011; 21: 550-3.

27. Zhou W, Wang K, Ruan W, Bo Z, Liu L, Cao Z, Chai L, Cao G. Higher methylation in genomic DNA indicates incomplete reprogramming in induced pluripotent stem cells. Cell Reprogram. 2013; 15: 92-9.

28. Chang G, Gao S, Hou X, Xu Z, Liu Y, Kang L, Tao Y, Liu W, Huang B, Kou X, Chen J, An L, Miao K, et al. Highthroughput sequencing reveals the disruption of methylation of imprinted gene in induced pluripotent stem cells. Cell Res. 2014; 24: 293-306.

29. Gao S, Tao L, Hou X, Xu Z, Liu W, Zhao K, Guo M, Wang H, Cai T, Tian J, Gao S, Chang G. Genome-wide gene expression analyses reveal unique cellular characteristics related to the amenability of HPC/HSCs into high-quality induced pluripotent stem cells. Stem Cell Res Ther. 2016; 7: 40 .

30. Gao S, Chang G, Tian J, Gao S, Cai T. Identification of the new gene Zrsrl to associate with the pluripotency state in induced pluripotent stem cells (iPSCs) using high throughput sequencing technology. Genom Data. 2014; 2 : 73-7.

31. Gao S, Zheng C, Chang G, Liu W, Kou X, Tan K, Tao L, Xu K, Wang H, Cai J, Tian J, Gao S. Unique features of mutations revealed by sequentially reprogrammed induced pluripotent stem cells. Nat Commun. 2015; 6: 6318.

32. da Rocha ST, Edwards CA, Ito M, Ogata T, Ferguson-Smith AC. Genomic imprinting at the mammalian Dlk1-Dio3 domain. Trends Genet. 2008; 24: 306-16.

33. Liu L, Luo GZ, Yang W, Zhao X, Zheng Q, Lv Z, Li W, Wu HJ, Wang L, Wang XJ, Zhou Q. Activation of the imprinted Dlk1-Dio3 region correlates with pluripotency levels of mouse stem cells. J Biol Chem. 2010; 285: 19483-90.

34. Bartolomei MS, Ferguson-Smith AC. Mammalian genomic imprinting. Cold Spring Harb Perspect Biol. 2011; 3.

35. Carey BW, Markoulaki S, Hanna JH, Faddah DA, Buganim Y, Kim J, Ganz K, Steine EJ, Cassady JP, Creyghton MP, Welstead GG, Gao Q, Jaenisch R. Reprogramming factor stoichiometry influences the epigenetic state and biological properties of induced pluripotent stem cells. Cell Stem Cell. 2011; 9: 588-98.

36. Li Z, Wang L, Wang Y, Liu L, Wang L, Li W, Zhou Q. Generation of an lncRNA Gtl2-GFP reporter for rapid assessment of pluripotency in mouse induced pluripotent stem cells. J Genet Genomics. 2015; 42: 125-8.

37. Rideout WM 3rd, Eggan K, Jaenisch R. Nuclear cloning and epigenetic reprogramming of the genome. Science. 2001; 293: 1093-8.

38. Watanabe A, Yamada Y, Yamanaka S. Epigenetic regulation in pluripotent stem cells: a key to breaking the epigenetic barrier. Philos Trans R Soc Lond B Biol Sci. 2013; 368: 20120292.

39. Wu H, Zhang Y. Tet1 and 5-hydroxymethylation: a genomewide view in mouse embryonic stem cells. Cell Cycle. 2011; 10: 2428-36.

40. Koh KP, Yabuuchi A, Rao S, Huang Y, Cunniff K, Nardone J, Laiho A, Tahiliani M, Sommer CA, Mostoslavsky G, Lahesmaa R, Orkin SH, Rodig SJ, et al. Tet1 and Tet2 regulate 5-hydroxymethylcytosine production and cell lineage specification in mouse embryonic stem cells. Cell Stem Cell. 2011; 8: 200-13.

41. Gao Y, Chen J, Li K, Wu T, Huang B, Liu W, Kou X, Zhang Y, Huang H, Jiang Y, Yao C, Liu X, Lu Z, et al. Replacement of Oct4 by Tet1 during iPSC induction reveals an important role of DNA methylation and hydroxymethylation in reprogramming. Cell Stem Cell. 2013; 12: 453-69. 
42. Miyoshi N, Kuroiwa Y, Kohda T, Shitara H, Yonekawa H, Kawabe T, Hasegawa H, Barton SC, Surani MA, KanekoIshino T, Ishino F. Identification of the Meg1/Grb10 imprinted gene on mouse proximal chromosome 11, a candidate for the Silver-Russell syndrome gene. Proc Natl Acad Sci U S A. 1998; 95: 1102-7.

43. Holt LJ, Siddle K. Grb10 and Grb14: enigmatic regulators of insulin action--and more? Biochem J. 2005; 388: 393-406.

44. Riedel H. Grb10 exceeding the boundaries of a common signaling adapter. Front Biosci. 2004; 9: 603-18.

45. Kabir NN, Kazi JU. Grb10 is a dual regulator of receptor tyrosine kinase signaling. Mol Biol Rep. 2014; 41: 1985-92.

46. Plasschaert RN, Bartolomei MS. Tissue-specific regulation and function of Grb10 during growth and neuronal commitment. Proc Natl Acad Sci U S A. 2015; 112: 6841-7.

47. Yan X, Himburg HA, Pohl K, Quarmyne M, Tran E, Zhang Y, Fang T, Kan J, Chao NJ, Zhao L, Doan PL, Chute JP. Deletion of the Imprinted Gene Grb10 Promotes Hematopoietic Stem Cell Self-Renewal and Regeneration. Cell Rep. 2016; 17: 1584-94.

48. Kou Z, Kang L, Yuan Y, Tao Y, Zhang Y, Wu T, He J, Wang J, Liu Z, Gao S. Mice cloned from induced pluripotent stem cells (iPSCs). Biol Reprod. 2010; 83: 238-43.

49. Yin R, Mao SQ, Zhao B, Chong Z, Yang Y, Zhao C, Zhang D, Huang H, Gao J, Li Z, Jiao Y, Li C, Liu S, et al. Ascorbic acid enhances Tet-mediated 5-methylcytosine oxidation and promotes DNA demethylation in mammals. J Am Chem Soc. 2013; 135: 10396-403.

50. Zhao B, Yang Y, Wang X, Chong Z, Yin R, Song SH, Zhao C, Li C, Huang H, Sun BF, Wu D, Jin KX, Song M, et al. Redox-active quinones induces genome-wide DNA methylation changes by an iron-mediated and Tet-dependent mechanism. Nucleic Acids Res. 2014; 42: 1593-605. 